\title{
Myoclonus of the Auricular Muscles as the Cause of Objective
}

\section{Tympanophonia}

\author{
Boiko NV1, Stagnieva IV1, Doykov I² and Vicheva $\mathrm{D}^{2 *}$ \\ ${ }_{1}^{1}$ Department of Otorhinolaryngology, Rostov State Medical University, Russia \\ ${ }^{2}$ Department of Otorhinolaryngology, Plovdiv Medical University, Bulgaria
}

*Corresponding author: Dilyana Vicheva, Department of Otorhinolaryngology,

Plovdiv Medical University, Bulgaria, Tel: +359 888223675; Email: vdilyana@gmail.com

\section{Editorial}

Volume 3 Issue 2

Received Date: July 17, 2018

Published Date: July 19, 2018

DOI: $10.23880 / 00 A J-16000173$

\section{Abstract}

The objective of the present article was to systematize the available data on the etiology, pathogenesis, clinical features, diagnostics and treatment of muscular tympanophonia. The common sourse of muscular tympanophonia is the tremor (myoclonus) of the soft palate or muscles of auditory ossicles. Sometimes this condition can be a consequence of myoclonus of the external ear muscles. Pharmacotherapy of muscular tympanophonia does not invariably result in the favourable outcome. The authors describe a rare observation of objective tympanophonia attributable to myoclonus of auricular muscle.

Keywords: Objective tympanophonia; Myoclonus

\section{Abbreviations: MEM: Middle Ear Myoclonus.}

\section{Introduction}

Tympanophony, or tinnitus, is a phantom auditory perception without any outer sound stimulation [1]. Tympanophony is subdivided into two categories: subjective and objectively perceived noises. Most patients suffer from subjective tympanophony since statistically it occurs to $5-15 \%$ of the population [2], while objective tympanophony is a much rarer phenomenon.

Objectively perceived noises can be of vascular and muscle origins. Vascular pulsating noise occurs with arteriovenous aneurysms, arteriosynus fistulas $[3,4]$, as well as middle ear paragangliomas [5]. Transitory vascular tympanophony may also be attributed to drug administration, arterial hypertension, anemia, or inter current diseases like browache [6].

Objective muscle tympanophony is based upon consensual spastic muscle activity (myoclonus) perceived as ear clicks. The PubMed database of 1955 to 2016 offers 104 publications describing cases of objective tympanophony caused by myoclonus of various muscle groups.

Muscle tympanophony typically results from palatal tremor (myoclonus) or middle ear myoclonus. However there are a few descriptions of tympanophony associated with eyelid movement [7], ear outer muscle myoclonus (M. auricularis anterior, superior, posterior), and head 


\section{Otolaryngology Open Access Journal}

muscle myoclonus (M. temporalis and M. occipitalis) [810].

Palatal myoclonus is manifested by palatal rhythmical, out-of-control contractions. Publications point out two types of myoclonus: symptomatic, conditioned by a brain bridge or tentorium bridge damage, and essential found in patients without any intracranial pathology $[11,12]$.

Palatal tremor clinically manifests itself as a sensation of palatal not-willed movements together with rhinolalia or without it (in $20 \%$ of patients), ear clicks (in $46.7 \%$ of patients), or both (in $33.3 \%$ of patients) [13]. When examined, $53.3 \%$ of patients show throat muscle movements synchronized with those of the palate [13]. Palatal tremor also takes place while the patient is asleep, yet disappears during swallowing and in the dorsal position. Objective tympanophony manifested as clicks resulting from palatal tremor is thought to be stipulated by secondary movements of the auditory tube walls [14].

Attempts to treat such patients with anxiolytic and anticonvulsive drugs, as well as surgical treatment and masking white noise application, appeared to be inefficient. Some researchers $[11,15,13]$ shared their positive experience of injecting botulinum toxin in case of palatal myoclonus. Botulinum toxin inhibits release of acetylcholine from presynaptic nerve terminals thus causing muscle "chemical denervation" which lasts several weeks. Botulinum toxin injection into the soft palate may cause side effects like hyponasal speech, velopharyngeal inadequacy resulting in driving swallowed food to the nasal cavity, dysphagia, as well as subjective noise gain in the opposite ear as a result of the unmasking effect. Generally such adverse events remit within 10-14 days. Side effect evidence may be minimized by target botulinum toxin injection into the area of maximum myoclonic activity under electromyography control $[15,16]$. The dose is to be individually chosen for each patient by the titration method $[11,15,13]$.

The botulinum toxin injection schedule depends on the patient's prevailing complaint: in case of tympanophony the medication is injected transorally into $\mathrm{m}$. tensor velipalatini in the lateral area of the palate medially towards the projection of pterygoid bone hook; in case of dominating sensation of palatal non-willed movements, the injection is made on both sides of the uvula [13]. The dose and the point of subsequent injections depend on the effect of the first injection and the area of dominating muscle contractions.

Middle ear myoclonus (MEM) is another possible cause of objective tympanophony. This term was offered to denote tinnitus resulting from the dysfunction of one or both end aural muscles: $\mathrm{m}$. tensor tympani and $\mathrm{m}$. stapedius. Tympanophony in case of middle ear myoclonus is characterized as: rhythmical, regular / irregular, long-lasting / episodic, unilateral / bilateral [17]. This type of pathology may be detected due to indicative clinical particulars (clicking sensation in the ear), as well as based upon the impedansometry and otomicroscopy data (in myoclonus m. tensor tympani).

Pathophysiological and acoustic mechanisms of middle ear myoclonus are undefined. Researchers have to give answers to a number of questions [18] does palatal myoclonus and middle ear myoclonus manifest a single disease, or are these different malconditions? Do ear clicks derive from palatal myoclonus (in other words, do they result from the strokes of the auditory tube walls), or do they result from the contraction of the muscle straining the eardrum? Can otostapes muscle contractions produce clicks in the ear?

Ellenstein A, et al. [17] believes that middle ear myoclonus may be explained by peritubal muscle myoclonus similar to essential palatal tremor. Meanwhile there exists a clinical study [19] where tympanophony caused by middle ear myoclonus was successfully stopped with a botulinum toxin sponge placed close to $\mathrm{m}$. stapedius sinew through the existing tympanic membrane perforation, which enables to associate typical muscle noise definitely with otostapes muscle myoclonus.

Difficulties in learning middle ear myoclonus are due to the fact that there are just miscellaneous descriptions of a few observations of this tympanophony variety (as a rule, 1-2 cases), which do not give a distinct description of the phenomenology, neither identify the diseased muscle [17].

The largest number of middle ear myoclonus cases are presented by Park SN, et al. [20] 58 patients observed between 2004 and 2011. These authors noted that the disease could be most often observed after stress or noise exposure (51.8\% and $27.6 \%$ correspondingly). Unlike subjectivity $M$. panophony, drug treatment of the analyzed group proved to be effective in $75 \%$ of cases [20], in these instances the patients were treated by anticonvulsive and muscle relaxant drugs.

If conservative treatment of intra-aural muscle myoclonus is ineffective, there is a possibility of surgical interference, i.e. selective tenotomy of the diseased muscle. Prior to the surgery the doctor has to differentiate stapedial muscle myoclonus and eardrum myoclonus. Hidaka $\mathrm{H}$, et al. [21] analyzed their own experience of 


\section{Otolaryngology Open Access Journal}

treating 23 cases of tympanophony caused by intra-aural muscle myoclonus, as well as literature data, and singled out those factors of diagnostic significance indicating tenotomy demand: facial nerve paresis, tympanophony provoking factors, myoclonus visualization during surgery. The first two factors are of special importance in the detection of indications for selective tenotomy $\mathrm{m}$. stapedius ( $\mathrm{p}<0.05$ and $\mathrm{p}<0.01$ correspondingly). One possible complication after $\mathrm{m}$. stapedius tenotomy is hyperacusia [22] or even phonophobia, which significantly deteriorates the patient's life quality.

Ear aurical muscle myoclonusis an extremely rare phenomenon. Publications define it with various terms: ear tic, moving ear syndrome, muscle dystonia, ear dyskinesia [23-27]. Ear aurical muscle myoclonus, as it appears from one of the titles, is often accompanied by its evident rhythmical movement, yet as a rule it disappears at bedtime and at breath hold [10].

Objectivity $M$. panophony treatment is a difficult process. One possible therapy for tympanophony is drug treatment, yet the assessment of its effectiveness is rather contradictory, and its evidential base is insufficient $[10,2]$.

Espinosa-Sánchez JM, et al. [2] in their review paper analyze the effectiveness of anaesthetic, anticonvulsant, antidepressant drugs, antihistamines, benzodiazines, diuretic drugs, corticosteroids to reduce tympanophony intensity. According to publications, intravenous administration of lidocaine leads to a short-term effect, however this method did not arouse widespread due to high possibility of adverse events. Cambamazepine and gabapentin may be rather effective in quite a number of myoclonus cases, yet controlled studies did not record any significant differences from placebo. Tricyclic antidepressants, serotonin reuptake inhibitors and benzodiazines neither demonstrated evidence-based effectiveness, although many patients state improvement of the emotional background as they take these medications.

What follows is our own clinical impression of a case of objective tympanophony caused by ear auricle myoclonus.

A 62 year old patient complained of clicking sensations in his right ear, synchronized with not-willed muscle contractions above the ear auricle and in the postaural area. These symptoms had started 6 months before and stopped at night time. The patient had had no head injuries, neither had he taken medications that could provoke myoclonus.
On examination: eardrums and epipharynx were without acute pathology. No asymmetry of contraction or twitching of palatal, throat and neck muscles, neither skull nerve dysfunction were revealed. As the ear clicks started, palpation revealed muscle activity in the postaural area and above the auricle. Electromyography showed short firings of spontaneous musculation lasting 50 to $100 \mathrm{~ms}$ in $\mathrm{m}$. auricularis posterior and $\mathrm{m}$. temporalis. Tone threshold audiometry showed1st degree bilateral sensorineural hearing loss. MRT revealed no pathology in brain stem structures. On neurologist's order the patient had been taking clonazepam and baclofen during 5 weeks, yet without any improvement. Judging from ineffectiveness of the previous treatment, we administered botulinum toxin injections according to the schedule proposed by Lee K [8] 10 units of botulinum toxin into m. auricularis posterior area and 10 units into m. temporalis area. After 8 days the patient stated there were no ear clicks. No side effects were noted.

Ear auricle myoclonus may be either an independent disease or a palatal tremor manifestation [28]. Since palatal tremor may spread to remote areas [29], each clinical event demands differential exclusion. However when examined, patient $\mathrm{K}$. did not show any signs of palatal tremor, and available publications do not offer descriptions of isolated myoclonus of ear auricle muscles in palatal myoclonus. The patient distinctly sensed convulsive twitches above the ear auricle and in the postaural area, which was confirmed by electromyography. Effectivity of botulinum toxin injection into the diseased muscles is another indirect support of the correct detection of the pathology location.

Thus, objective tympanophony caused by muscle myoclonus possesses sharply defined clinical particulars: it may be long-lasting or episodic, unilateral or bilateral, sensed by the patient as ear clicks, and it is never tonal. A noise of the kind can often be heard by the people around. In diagnosticating objective muscle tympanophony importance should be attached to visual detection of rhythmical, out-of-control contractions of separate muscle groups (palatal tremor, aurical movements), otomicroscopy and impedansometry (for middle ear myoclonus), electromyography, brain MRT.

\section{References}

1. Jastreboff PJ (1990) Phantom auditory perception (tinnitus): mechanisms of generation and perception. Neurosci Res 8(4): 221-254. 


\section{Otolaryngology Open Access Journal}

2. Espinosa-Sánchez JM, Heitzmann-Hernández T, López-Escámez JA (2014) Pharmacotherapy for tinnitus: much ado about nothing. Rev Neurol 59(4): 164-174.

3. Boiko NV (2017) Tinnitus: Algorithm of diagnostics and clinical management. Zhurnal Nevrol Psihiatr Im SS Korsakova 117(9): 88-93.

4. Kolesnikov VN, Boiko NV, Pisarenko EA (2011) Surgical removal of tympanic paragangliomas with sound-transmitting structures of the middle ear preservation. Rossiiskaya Otorinolaringol 55(6): 6977.

5. Madania G, Connorb SEJ (2009) Imaging in pulsatile tinnitus. Clinical Radiology 64(3): 319-328.

6. Srirompotong S, Saeseow P, Kharmwan S (2007) Ear wiggling tics: treatment with botulinum toxin injection. Eur Arch Otorhinolaryngol 264(4): 385387.

7. Gandhi SE, Kellett MW, Kobylecki C (2014) Botulinum toxin-responsive ear myoclonus following otitis externa. Letter to the Editor. Parkinsonism and Related Disorders 20(2): 265-266.

8. Lee K, Chang J, Park S, Im GJ, Choi HJ, et al. (2014) Bilateral muscular tinnitus due to myoclonus of extrinsic auricular muscles. Auris Nasus Larynx 42(2): 173-175.

9. Caviness JN (2009) Pathophysiology and Treatment of Myoclonus. Neurol Clin 27(3): 757-777.

10. Kim TH, Jang HJ, Park SH, Nam SI (2015) Objective Tinnitus Concomitant with Eye Blinking: A Case Report Audiol Otol 19(2): 101-103.

11. Penney SE, Bruce IA, Saeed SR (2006) Botulinum toxin is effective and safe for palatal tremor: a report of five cases and a review of the literature. J Neurol 253(7): 857-860.

12. Zadikoff C, Lang AE, Klein C (2006) The 'essentials' of essential palatal tremor: a reappraisal of the nosology. Brain 129(4): 832-840.

13. Sinclair CF, Gurey LE, Blitzer A (2014) Palatal myoclonus: algorithm for management with botulinum toxin based on clinical disease characteristics. Laryngoscope 124(5): 1164-1169.
14. MacDonald JT (2006) Objective Tinnitus Due to Essential Palatal Tremor in a 5-Year-Old. Pediatr Neurol 36(3): 175-176.

15. Anis MM, Pollak N (2013) Treatment of palatal myoclonus with botulinum toxin injection. Case Rep in Otolaryngol 2013: 231505.

16. Wan TK, Chen JT, Wang PC (2013) EMG-guided salpingopharyngeus Botox injection for palatal myoclonus. B-ENT 9(1): 67-69.

17. Ellenstein A, Yusuf N, Hallett M (2013) Middle ear myoclonus: two informative cases and a systematic discussion of myogenic tinnitus. Tremor and Other Hyperkinet Mov (NY) 3.

18. Oliveira CA, Negreiros J, Cavalcante IC, Bahmad F, Venosa AR (2003) Palatal and middle-ear myoclonus: a cause for objective tinnitus. Int Tinnitus J 9(1): 37 41.

19. Liu HB, Fan JP, Lin SZ, Zhao SW, Lin Z (2011) Botox transient treatment of tinnitus due to stapedius myoclonus: Case report. Clin Neurol Neurosurg 113(1): 57-58.

20. Park SN, Bae SC, Lee GH, Song JN, Park KH, et al. (2013) Clinical characteristics and therapeutic response of objective tinnitus due to middle ear myoclonus: a large case series. Laryngoscope 123(10): 2516-2520.

21. Hidaka H, Honkura Y, Ota J, Gorai S, Kawase T, et al. (2013) Middle ear myoclonus cured by selective tenotomy of the tensor tympani: strategies for targeted intervention for middle ear muscles. Otol Neurotol 34(9): 1552-1558.

22. Silverstein H (1998) Laser stapedotomy minus prosthesis (laser STAMP): a minimally invasive procedure. Am J Otol 19(3): 277-282.

23. Chaudhuri KR, Leigh PN, Gibb WR (1996) The moving ear syndrome: a focal dyskinesia. J Neurol Neurosurg Psychiatry 60(1): 106-110.

24. Supaporn S, Patachareeporn S, Samerduan K, Somchai S (2007) Ear wiggling tics: treatment with botulinum toxin injection. Eur Arch Otorhinolaryngol 264(4): 385-387.

25. Alonso-Navarro H, Puertas I, Cabrera-Valdivia F, de Toledo-Heras M, Garcia- Albea E, et al. (2007) 


\section{Otolaryngology Open Access Journal}

Posterior auricular muscle 'dystonia'. Eur J Neurol 14(7): 14-15.

26. Godeiro-Junior C, Felicio AC, Felix EP, Manzano GM, Silva SM, et al. (2008) Moving ear syndrome: the role of botulinum toxin. Mov Disord 23(1): 122-124.

27. Boiko NV (2017) Objective tympanophonia caused by myoclonus of the auricular muscles. Vestnik Otorinolaringol 82(3): 80-83.
28. Kirk A, Heilman KM (1991) Auricular myoclonus. Can J Neurol Sci 18(4): 503-504.

29. Riley HA, Brock S (1933) Rhythmic myoclonus of the muscles of the palate, pharynx, larynx and other regions: a clinical report of three cases. Arch Neurol Psychiatry 29(4): 726-741. 